\title{
Editorial
}

\section{Nanomaterials and the Environment}

\author{
Prashant Kumar, ${ }^{1}$ Arun Kumar, ${ }^{2}$ Teresa Fernandes, ${ }^{3}$ and Godwin A. Ayoko ${ }^{4}$ \\ ${ }^{1}$ Department of Civil \& Environmental Engineering, Faculty of Engineering and Physical Sciences, \\ University of Surrey, Guildford GU2 7XH, UK \\ ${ }^{2}$ Department of Civil Engineering, Indian Institute of Technology New Delhi, Hauz Khas, New Delhi 110016, India \\ ${ }^{3}$ School of Life Sciences, Heriot-Watt University, Edinburgh EH14 4AS, UK \\ ${ }^{4}$ Nanotechnology and Molecular Sciences, School of Chemistry, Physics and Mechanical Engineering, \\ Queensland University of Technology, Brisbane, QLD 4001, Australia \\ Correspondence should be addressed to Prashant Kumar; p.kumar@surrey.ac.uk
}

Received 20 August 2014; Accepted 20 August 2014; Published 3 September 2014

Copyright (C) 2014 Prashant Kumar et al. This is an open access article distributed under the Creative Commons Attribution License, which permits unrestricted use, distribution, and reproduction in any medium, provided the original work is properly cited.

The subject of nanomaterials is very topical, with advances in knowledge made at a very rapid pace, making dissemination of this new knowledge a need of the hour. Although it is widely recognised that nanotechnology is playing a key role in many areas of societal endeavour, it is still unclear what risks certain nanomaterials may pose to humans and the environment. While nanotechnology has brought enormous benefits to humankind, its impact on human health and the environment is yet to be fully understood.

Nanomaterials are considered as emerging environmental contaminants. Their origin can be natural [1], incidental [2], or from manufacturing processes [3]. Incidental nanomaterials are those generated as side products of anthropogenic processes $[4,5]$, whereas manufactured nanomaterials are deliberately produced with specific properties [3, 6]. Exposure to both types is currently being investigated and these may enter air, water, and soil media from a range of routes. Physicochemical and biological transformations make nanomaterials potentially highly reactive in both environmental and biological systems, which may alter their fate, dispersion, and toxicity compared with their larger counterparts $[7,8]$.

Recent years have seen an enormous increase in the number of publications, indicating an exponential increase over the past decade in nanomaterial-related research, in terms of manufacturing, applications, exposure, and hazard (Figure 1). These studies have focused on various aspects of nanomaterials, such as their novel applications as adsorbents, ion exchangers and disinfectants in water and air for removing ions, and organic compounds and pathogens $[9,10]$, as well as assessing risks associated with them to human health, ecology, and environment [11]. This special issue was designed to highlight recent advances in the area of nanosafety as well as present studies on the application of nanomaterials for environmental remediation, such as removal of ions and organic compounds from aqueous and air media. We believe that the articles published in this special issue are of great interest to scientists engaged in crossdisciplinary research and other stakeholders alike.

Given the highly interdisciplinary nature of the topic, a broad range of researchers from the scientific community were invited to contribute original research articles as well as review articles that could stimulate the continuing efforts to understand the advances in nanomaterial characterisation, emissions, transformation, dispersion, fate, and effects in different environmental compartments (air, water, and soil). A particular focus of the issue was on articles that can deal with their environmental and health impacts, and the implications for policy and regulations for both the indoor and outdoor environments.

A broad range of the scientific community participated in this special issue, starting from material scientists working on the development of novel materials to those using them in various applications. Scientists involved in carrying out environmental and health impact assessment of nanomaterials made their contributions too. The other very interested community participating in this special issue was 


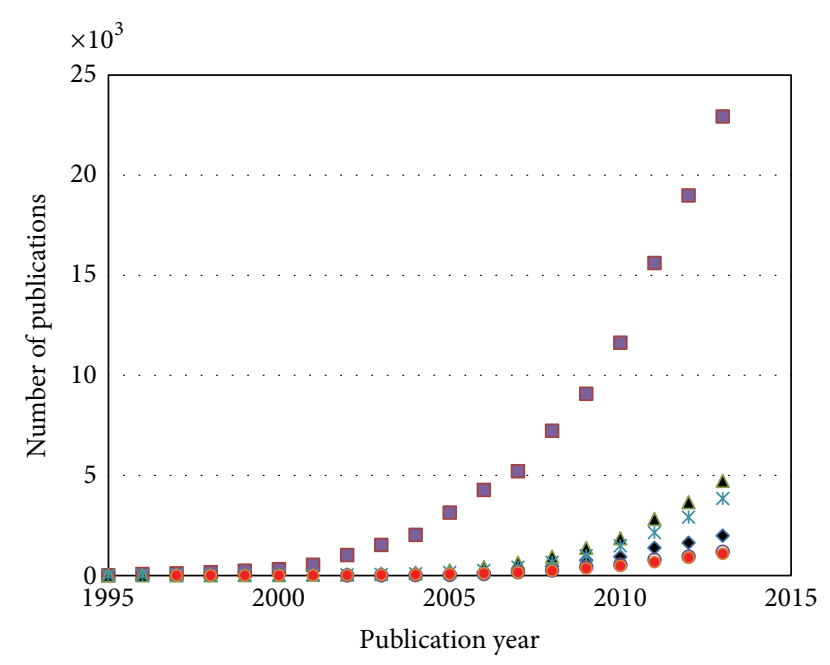

- Nanomaterial

- Nanomaterials risk

- Nanomaterials environment risk

* Nanomaterials water treatment

^ Nanomaterials environment - Nanomaterials air treatment

FIgURE 1: Trend of published scientific articles over time in various nanomaterials-related areas (data obtained from Scopus on July 4, 2104 , using keywords mentioned in legends of this figure).

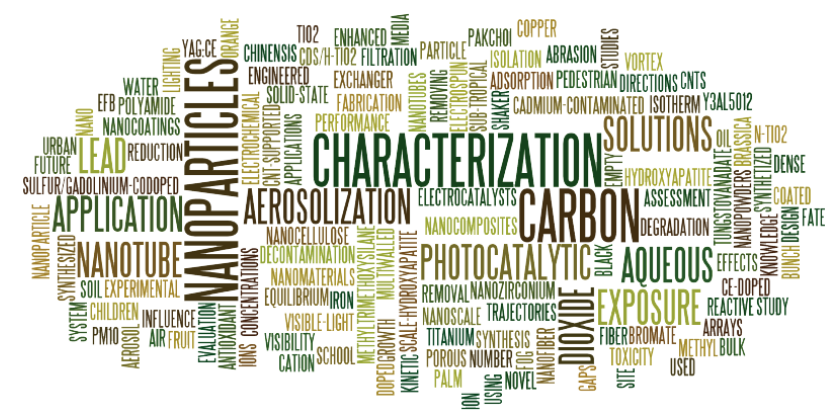

FIGURE 2: Frequency distribution of words used in titles of articles published in this special issue. Increasing size of words shows their more frequent use in published titles.

those working in the area of environmental pollution (air, water, and soil), their emissions, dispersion, exposure, and fate, given that there is still lack of studies in the field of the fate of nanomaterials in different environmental media. Very importantly, the published articles have relevance to regulatory authorities and policy makers in order to manage and mitigate environment and public health risks associated with nanomaterials.

This special issue received a very good response from authors and a good number of research articles were submitted. From those, about $60 \%$ were accepted for publication. These articles covered a broad variety of topics related to nanomaterials in air and water environments, exposure, and risks of nanomaterials, whereas some were technologyfocused. A reflection of the topic areas covered is seen in Figure 2 which shows the frequency distribution of the words used in titles of published articles.
There were two submissions related to particulate matter and exposure risks in urban atmospheric environments. The work of J. Garcia et al. used computational fluid dynamics (CFD) simulations to study the effect of different pedestrian trajectories on the exposure of school children to $\mathrm{PM}_{10}$ $(<10 \mu \mathrm{m}$ in aerodynamic diameter) concentrations in an urban street canyon in city of Barreiro, Portugal. Urban street canyons act as pollution "hot-spots" due to limited dispersion of pollution emitted by road vehicles. The work concluded that modelling can help with prediction of hotspots by using atmospheric data. Results from this modelling can help minimise exposure in busy street canyons by careful selection of the best pedestrian paths. The contribution from M. Mohan and S. Payra investigated the relationships between ambient aerosol number concentrations (ANC) and meteorological parameters to study the fog conditions in Delhi. Their findings suggested a threshold minimum value of ANC for foggy conditions and obtained a relationship in power form between the ANC and visibility.

N. S. Lani et al. isolated nanocellulose from empty fruit bunch (EFB) fiber and studied their reinforcing effect in polyvinyl alcohol (PVA)/starch blend films. This work reported that PVA/starch films reinforced with nanocellulose fiber possess significantly improved mechanical properties compared to unreinforced film, and this nanocomposite has good water resistance and biodegradability, which highlights its potential in future applications.

J. Matulevicius et al. investigated electrospun polyamide 6 (PA 6) and polyamide 6/6 (PA 6/6) nanofibers for air filtration applications. Experimental results enabled the response surfaces for desirable characteristics of useful nanofiber filters to be derived while modelling results showed that electrospun polyamide $6 / 6$ fibre media derived from $8 \%(\mathrm{w} / \mathrm{vol})$ solutions of formic acid/acetic acid ( $3: 2 \mathrm{vol} / \mathrm{vol})$ have the smallest fibre diameters. Therefore, they had the highest filtration efficiencies (up to 90\%) and displayed the best filtration quality factors (up to $0.0749 \mathrm{~Pa}^{-1}$ ). The paper highlights the potential role of modelling in predicting suitable characteristics of fibers for air filtration applications.

A. Zeino et al. studied the removal of bromate from drinking water by different types of carbon nanotubes (CNTs). These authors compared raw CNTs and CNTs oxidised with nitric acid, with CNTs impregnated with Fe. The latter CNTs had higher adsorption capacities. Nitric acid was found to be a better acid for the adjustment of the $\mathrm{pH}$ and the adsorption capacities of all CNTs decreased with adsorbent dose and $\mathrm{pH}$ of the solution but increased with the bromate concentration.

The work of Sk. S. Hossain et al. experimentally investigated copper loaded CNTs as electrocatalysts for the electrochemical reduction of carbon dioxide. Their results found that copper loaded CNTs ( $20 \%$ by weight) showed maximum activity among other catalysts tested, and the faradaic efficiency for methanol formation was estimated to be $38.5 \%$.

E. S. Agorku et al. reported the use of various sulfur/ gadolinium-codoped $\mathrm{TiO}_{2}$ nanoparticles for the visible light photocatalytic degradation of indigo carmine as a model organic pollutant. Appreciable visible light degradation was 
observed in all cases, with $\mathrm{TiO}_{2}-\mathrm{S} / \mathrm{Cd}^{3+}$ showing more activity than commercial $\mathrm{TiO}_{2}$. The photocatalytic efficacy of codoped $\mathrm{TiO}_{2}$ nanoparticles increased with the percentage of $\mathrm{Cd}^{3+}$ up to $0.6 \% \mathrm{Cd}^{3+}$, with $\mathrm{TiO}_{2}-\mathrm{S} / \mathrm{Cd}^{3+}\left(0.6 \% \mathrm{Cd}^{3+}\right)$ showing the highest activity and degrading indigo carmine completely in 50 minutes.

$\mathrm{N}$. Shandilya et al. evaluated the release of $\mathrm{TiO}_{2}$ nanoparticles from two commercial photocatalytic nanocoatings using abrasion tests. One of the nanocoatings inhibited the release of nanoparticles while the other did not. No free $\mathrm{TiO}_{2}$ nanoparticles were released from either of the nanocoatings. The study introduced two particle release parameters that can be used to assess the tendency of nanocoatings to hold or release particles. O. L. C. Le Bihan et al. reported the aerosolization of a multiwalled CNT, by a vortex shaker. Their aims were to develop and evaluate vortex shaker techniques as a tool for the determination of the exposure potential to suspensions of inhalable particles and powders from nanomaterials. The study demonstrated that the geometry of the device and speed of agitation influenced the experimental outcome and concluded that although the method has the potential to be used for dustiness assessments further evaluation would be required before it can be routinely used for toxicological investigations.

$\mathrm{X}$. Zhou et al. fabricated and characterised $\mathrm{CdS} / \mathrm{H}-\mathrm{TiO}_{2}$ nanotube (TNT) arrays to investigate their photocatalytic properties during degradation of a methyl orange dye. The photodegradation of methyl orange was carried out under visible-light irradiation. They found that the CdS/H-TNTs exhibited greater efficiency than the H-TNTs and pure TNTs. In particular, the $\mathrm{CdS} / \mathrm{H}$-TNTs gave $88.7 \%$ decoloration rate compared to pure TNTs with only $6.4 \%$ decoloration rate. Their work also showed that the CdS/H-TNTs were approximately 13-times more effective than pure TNTs under visible light, indicating that these TNTs could successfully be used for decomposing methyl orange from water.

D. N. Chung et al. synthesised Ce-doped Y3Al5O12 (YAG: Ce) nanopowders using a sol-gel low temperature combustion method, followed by thermal annealing and used for solid-state lighting. They found that the white light emitting diodes (WLEDs) made from the blue light emitting diode (LED) chip coated with the nano-YAG: $\mathrm{Ce}+\mathrm{MEH}-$ PPV composite epoxy exhibited white light with a broad band luminescent spectrum and a high colour rending index. These findings indicated potential application of the prepared nanostructured YAG: Ce phosphor in energy-efficient solidstate lighting as well as in organic composite solar cells. These findings also indicate their application for the enrichment of uniform inorganic nanoparticles.

A few articles were submitted on nanomaterials used as adsorbents and ion exchange materials for removing dyes, metal ions, and inorganic ions from water. These studies showed that removal of ions and dyes from water using nanomaterial-based adsorbent and ion exchange material is a suitable and very promising approach. For example, $\mathrm{M}$. A. Shaheed and F. H. Hussein studied adsorption of reactive black 5 on synthesized titanium dioxide nanoparticles using equilibrium isotherm and kinetic studies that $\mathrm{TiO}_{2}-\mathrm{NPs}$ could be a promising adsorbent as it showed removal of $\mathrm{RB}$ 5 from aqueous solutions through a chemical adsorption method. The study found a Langmuir monolayer adsorption capacity of $88.495 \mathrm{mg} / \mathrm{g}$ at $\mathrm{pH} 5.5$ and $30^{\circ} \mathrm{C}$. M. F. Elkady et al. assessed the potential of synthesized nanozirconium tungstovanadate as cation exchanger and tested it in the removal of lead ions from water. They noticed $96 \%$ removal of lead ions from water and showed that the new material can act as cation exchanger (ion exchange capacity = 2.5 milliequivalent/g). C. S. Ciobanu et al. synthesized porous methyltrimethoxysilane coated nanoscale-hydroxyapatite and investigated its potential for removal of lead ions from aqueous solutions. This study characterized the prepared material using X-ray diffraction (XRD), Fourier transform infrared spectroscopy (FTIR), and scanning electron microscopy (SEM) equipped with an energy dispersive $\mathrm{X}$-ray spectrometer (EDS) and then studied lead removal in adsorption studies at different solution $\mathrm{pH}$ values. They noticed that at $\mathrm{Pb}$ concentrations from $0.5 \mathrm{~g} / \mathrm{L}$ to $1.5 \mathrm{~g} / \mathrm{L}$, the removal efficiency reached nearly $100 \%$ (i.e., complete removal). In general, removal was found to be higher in acidic $\mathrm{pH}$ conditions, which decreased as solution $\mathrm{pH}$ increased.

A few submissions presented the effect of nanomaterials on growth of plants and metals uptake by plants. This topic is particularly interesting given the fact that there are still very few studies assessing the effects of nanomaterials on soil systems, and particularly on plants. For example, Z. Li and J. Hunag studied effect of the nanoparticle hydroxyapatite (nHAP) on the growth and antioxidant system in Pakchoi (Brassica chinensis L.) from cadmium-contaminated soil by using different nHAP concentrations. Results indicated that increasing levels of nHAP led to improved plant growth and reduced Cd uptake by the plant. Also, exposure of higher nHAP levels resulted in an increase in levels of chlorophyll and vitamin $C$ and decrease in the level of malondialdehyde (MDA) in plant shoots. The findings of their study indicated that nHAP can be used to reduce the uptake of Cd by Pakchoi from Cd-contaminated soil which reduces exposure risk in higher food web levels.

The team of guest editors also contributed a comprehensive review article. This article presented a comprehensive summary of state of the art on the fate, exposure, and toxicity of engineered nanomaterials in air and water environments, highlighting research gaps and suggesting directions for future research. The review recognised the need to develop a combination of different analytical methods for determining nanomaterial number and mass concentration, conducting toxicity studies, obtaining relevant data for developing quantitative nanostructure toxicity relationships (QNTR), and initiating efforts to formulate guidelines for the regulation of ENMs in the environment.

Overall, we believe that the collection of these scientific articles provides a solid contribution to the understanding of nanomaterials and their impacts on the environment. Research findings from the published work will be useful for material scientists, environmental scientist and engineers, risk assessors, and regulators for addressing nanomaterialrelated issues in the environment. 


\title{
Acknowledgments
}

The team of guest editors thank all the authors who have contributed articles to this special issue for the Journal of Nanomaterials. We also thank the reviewers for their constructive and insightful comments that made the completion of this issue possible.

\author{
Prashant Kumar \\ Arun Kumar \\ Teresa Fernandes \\ Godwin A. Ayoko
}

\section{References}

[1] P. Kumar, A. Robins, S. Vardoulakis, and R. Britter, "A review of the characteristics of nanoparticles in the urban atmosphere and the prospects for developing regulatory controls," Atmospheric Environment, vol. 44, no. 39, pp. 5035-5052, 2010.

[2] P. Kumar, P. Fennell, and A. Robins, "Comparison of the behaviour of manufactured and other airborne nanoparticles and the consequences for prioritising research and regulation activities," Journal of Nanoparticle Research, vol. 12, no. 5, pp. 1523-1530, 2010.

[3] J. R. Peralta-Videa, L. Zhao, M. L. Lopez-Moreno, G. de la Rosa, J. Hong, and J. L. Gardea-Torresdey, "Nanomaterials and the environment: a review for the biennium 2008-2010," Journal of Hazardous Materials, vol. 186, no. 1, pp. 1-15, 2011.

[4] F. Azarmi, P. Kumar, and M. Mulheron, "The exposure to coarse, fine and ultrafine particle emissions from concrete mixing, drilling and cutting activities," Journal of Hazardous Materials, vol. 279, pp. 268-279, 2014.

[5] P. Kumar, L. Pirjola, M. Ketzel, and R. M. Harrison, "Nanoparticle emissions from 11 non-vehicle exhaust sources: a review," Atmospheric Environment, vol. 67, pp. 252-277, 2013.

[6] P. Kumar, A. Kumar, and J. R. Lead, "Nanoparticles in the Indian environment: known, unknowns and awareness," Environmental Science and Technology, vol. 46, no. 13, pp. 7071-7072, 2012.

[7] Y. Ju-Nam and J. R. Lead, "Manufactured nanoparticles: an overview of their chemistry, interactions and potential environmental implications," Science of the Total Environment, vol. 400, no. 1-3, pp. 396-414, 2008.

[8] G. V. Lowry, K. B. Gregory, S. C. Apte, and J. R. Lead, "Transformations of nanomaterials in the environment focus issue," Environmental Science and Technology, vol. 46, no. 13, pp. 68916892, 2012.

[9] M. M. Khin, A. S. Nair, V. J. Babu, R. Murugan, and S. Ramakrishna, "A review on nanomaterials for environmental remediation," Energy and Environmental Science, vol. 5, no. 8, pp. 8075-8109, 2012.

[10] T. Pradeep, "Noble metal nanoparticles for water purification: a critical review," Thin Solid Films, vol. 517, no. 24, pp. 6441-6478, 2009.

[11] K. D. Grieger, I. Linkov, S. F. Hansen, and A. Baun, "Environmental risk analysis for nanomaterials: review and evaluation of frameworks," Nanotoxicology, vol. 6, no. 2, pp. 196-212, 2012. 

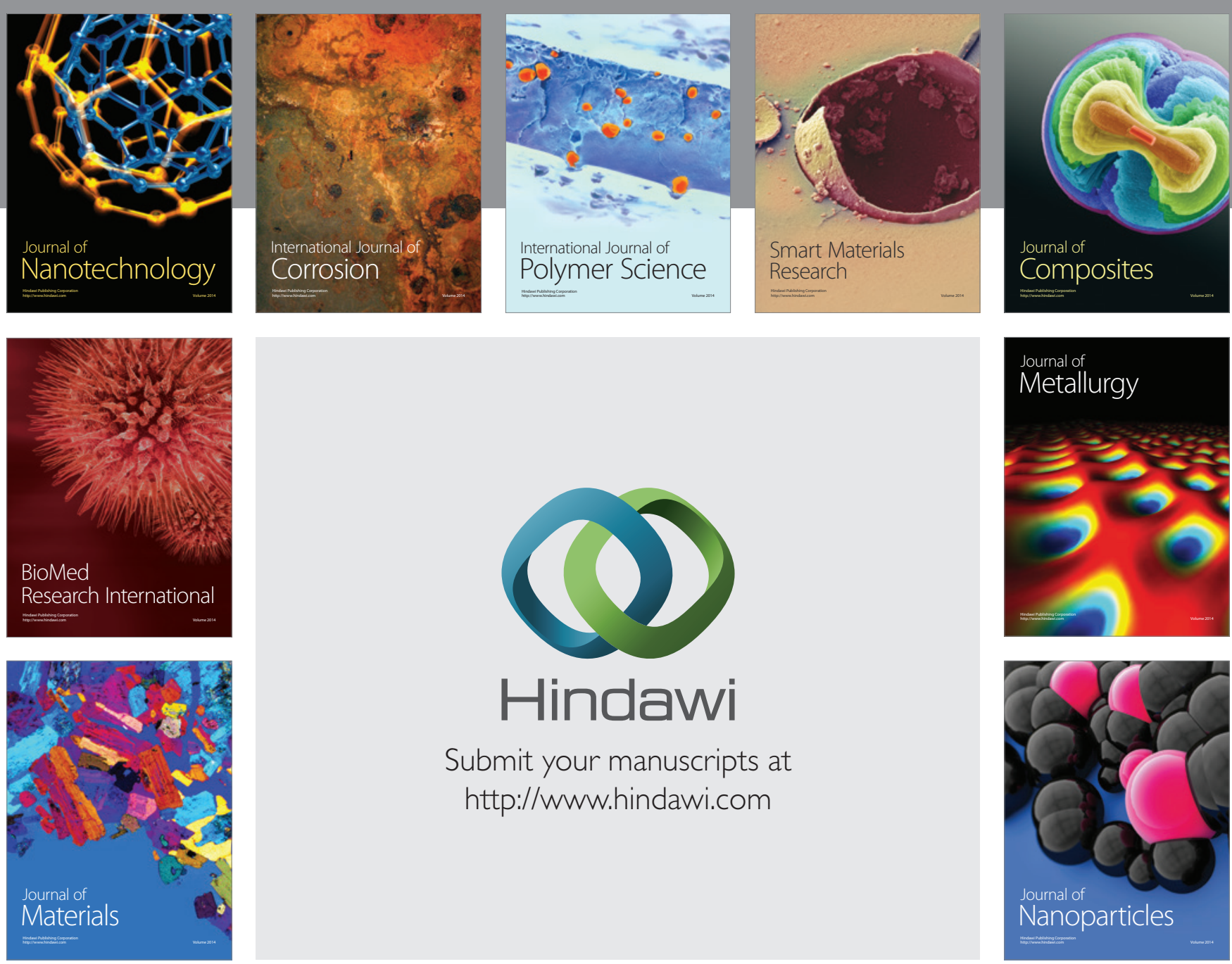

Submit your manuscripts at http://www.hindawi.com
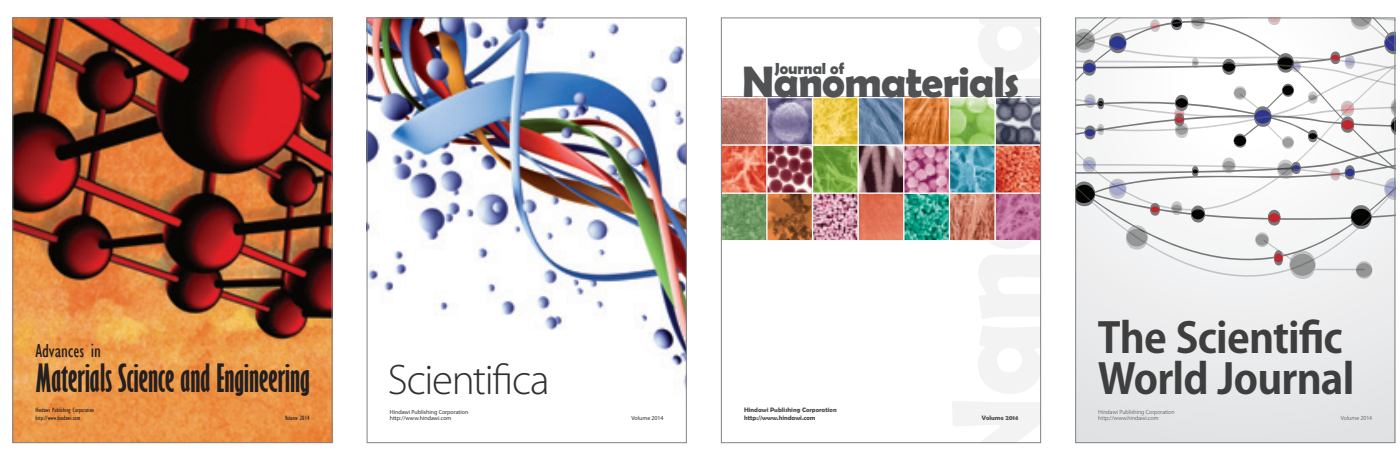

\section{The Scientific World Journal}
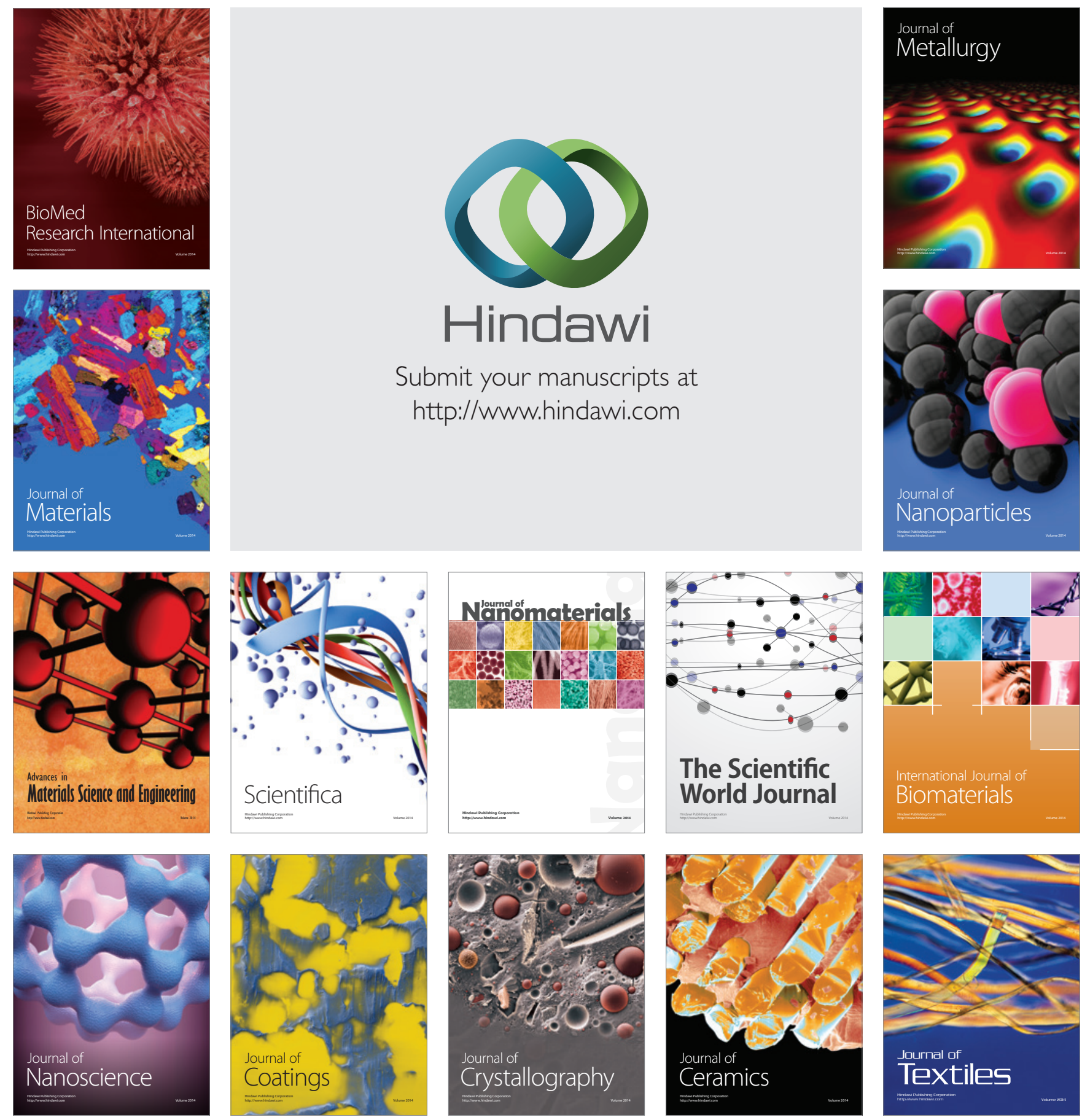